DAMTP-1998-160

gr-qc/9812032

\title{
Bounds on negative energy densities in static space-times
}

\author{
Christopher J. Fewsterf \\ Department of Mathematics, University of York, Heslington, York YO10 5DD, England \\ Edward Teoll \\ Department of Applied Mathematics and Theoretical Physics, University of Cambridge, \\ Silver Street, Cambridge CB3 9EW, England \\ Department of Physics, National University of Singapore, Singapore 119260
}

\begin{abstract}
Certain exotic phenomena in general relativity, such as backward time travel, appear to require the presence of matter with negative energy. While quantum fields are a possible source of negative energy densities, there are lower bounds - known as quantum inequalities - that constrain their duration and magnitude. In this paper, we derive new quantum inequalities for scalar fields in static space-times, as measured by static observers with a choice of sampling function. Unlike those previously derived by Pfenning and Ford, our results do not assume any specific sampling function. We then calculate these bounds in static three- and four-dimensional Robertson-Walker universes, the de Sitter universe, and the Schwarzschild black hole. In each case, the new inequality is stronger than that of Pfenning and Ford for their particular choice of sampling function.
\end{abstract}

*Electronic address: cjf3@york.ac.uk

${ }^{\dagger}$ Electronic address: E.Teo@damtp.cam.ac.uk 


\section{Introduction}

In recent years, there has been much interest in various exotic solutions of general relativitysuch as traversable wormholes [1, 2], the Alcubierre "warp drive" [3], and the Krasnikov "tube" [䧃]-that permit hyperfast or backward time travel. However, these space-times without exception require the presence of matter which possess negative energy densities [5, 6, 7, 8], and hence violate the standard energy conditions.

Now, it is well-known that quantum field theory, unlike classical physics, allows energy density to be unboundedly negative at a point in space-time [9]. Should the theory place no restrictions on this negative energy, quantum fields could be used to produce gross macroscopic effects such as those mentioned above, or even a violation of cosmic censorship or the second law of thermodynamics. It is therefore important to have a quantitative handle on the permitted amount of negative energy in a neighbourhood of a space-time point.

Ford and Roman [10, 11] have found inequalities which constrain the duration and magnitude of negative energy densities for quantised free, real scalar fields in Minkowski space. They show that a static observer, who samples the energy density by time-averaging it against the Lorentzian function

$$
f(t)=\frac{t_{0}}{\pi} \frac{1}{t^{2}+t_{0}^{2}}
$$

obtains a result which is bounded from below by a negative quantity depending inversely on the characteristic timescale $t_{0}$. For example, in the case of a massless scalar field in four dimensions, the renormalised energy density in any quantum state satisfies

$$
\rho \geq-\frac{3}{32 \pi^{2} t_{0}^{4}} .
$$

This means the more negative the energy density that is present in an interval, the shorter the duration of this interval must be. Thus, this "quantum inequality" - in a way reminiscent of the uncertainty principle of quantum mechanics凹 - serves to limit any large-scale, long-time occurrence of negative energy. In the infinite sampling time limit $t_{0} \rightarrow \infty$, it reduces to the usual averaged weak energy condition (for quantum fields [12, 13]).

Eveson and one of the present authors [14] have recently presented a different derivation of the quantum inequalities for a massive scalar field in $n$-dimensional Minkowski space (with $n \geq 2$ ). The method used is straightforward - involving only the canonical commutation

\footnotetext{
${ }^{1}$ However, the derivation of the quantum inequalities does not depend on any putative time-energy uncertainty principle.
} 
relations and the convolution theorem of Fourier analysis - and has the virtue of being valid for any smooth, non-negative and even sampling function decaying sufficiently quickly at infinity. Furthermore, the resulting bounds turn out to be stronger than those obtained by Ford and Roman [10, 11] when the Lorentzian sampling function is applied.

In the present paper, we extend this method to derive quantum inequalities for scalar fields in generally curved but static space-times using arbitrary smooth, non-negative (although not necessarily even, as assumed in [14]) sampling functions of sufficiently rapid decay. We obtain a lower bound on the averaged normal-ordered energy density in the Fock space built on the static vacuum in terms of the appropriate mode functions. Since the normalordered energy density in a given state is the difference between the renormalised energy density in this state and the (generally nonzero and potentially negative) renormalised energy density of the static vacuum, our bound also constrains the renormalised energy density (cf. [12]).

We apply our bound to several examples where the bound can be explicitly evaluated, namely the three- and four-dimensional Robertson-Walker universes, the de Sitter universe, and the Schwarzschild black hole. In all these cases, we obtain bounds which are up to an order of magnitude stronger than those previously derived by Pfenning and Ford [12, 13, 15] for the specific sampling function they used.

\section{Derivation of the quantum inequality}

We shall consider $n+1$-dimensional space-times that are globally static, with time-like Killing vector $\partial_{t}$. The metric of such a space-time takes the general form

$$
\mathrm{d} s^{2}=-\left|g_{t t}(\mathbf{x})\right| \mathrm{d} t^{2}+g_{i j}(\mathbf{x}) \mathrm{d} x^{i} \mathrm{~d} x^{j},
$$

where $\mathbf{x}=\left(x^{1}, x^{2} \ldots, x^{n}\right)$ and $i, j=1,2, \ldots, n$. The equation of a free, real scalar field $\phi$ of mass $\mu \geq 0$ in this space-time is

$$
-\frac{1}{\left|g_{t t}\right|} \partial_{t}^{2} \phi+\nabla^{i} \nabla_{i} \phi-\mu^{2} \phi=0 .
$$

Suppose it admits a complete, orthonormal set of positive frequency solutions. We write these mode functions as

$$
f_{\lambda}(t, \mathbf{x})=U_{\lambda}(\mathbf{x}) \mathrm{e}^{-i \omega_{\lambda} t}
$$


where $\lambda$ denotes the set of quantum numbers needed to specify the mode (which may be continuous or discrete). A general quantum scalar field can then be expanded as

$$
\phi=\sum_{\lambda}\left(a_{\lambda} f_{\lambda}+a_{\lambda}^{\dagger} f_{\lambda}^{*}\right)
$$

in terms of creation and annihilation operators $a_{\lambda}^{\dagger}, a_{\lambda}$ obeying the canonical commutation relations

$$
\left[a_{\lambda}, a_{\lambda^{\prime}}^{\dagger}\right]=\delta_{\lambda \lambda^{\prime}} \mathbb{1}, \quad\left[a_{\lambda}, a_{\lambda^{\prime}}\right]=\left[a_{\lambda}^{\dagger}, a_{\lambda^{\prime}}^{\dagger}\right]=0
$$

and which generate the Fock space built on the static vacuum state $|0\rangle$. We shall be interested in the energy density of $\phi$ along the world-line $x^{\mu}(t)=\left(t, \mathbf{x}_{\mathbf{0}}\right)$ of a static observer, with $\mathbf{x}_{\mathbf{0}}$ kept fixed. If the field is in a normalised quantum state $|\psi\rangle$, the normal-ordered energy density as measured by such an observer at time $t$ is 12, 13.

$$
\begin{aligned}
\left\langle: T_{\mu \nu} u^{\mu} u^{\nu}:\right\rangle=\operatorname{Re} \sum_{\lambda, \lambda^{\prime}} & \left\{\frac{\omega_{\lambda} \omega_{\lambda^{\prime}}}{\left|g_{t t}\right|}\left[U_{\lambda}^{*} U_{\lambda^{\prime}}\left\langle a_{\lambda}^{\dagger} a_{\lambda^{\prime}}\right\rangle \mathrm{e}^{i\left(\omega_{\lambda}-\omega_{\lambda^{\prime}}\right) t}-U_{\lambda} U_{\lambda^{\prime}}\left\langle a_{\lambda} a_{\lambda^{\prime}}\right\rangle \mathrm{e}^{-i\left(\omega_{\lambda}+\omega_{\lambda^{\prime}}\right) t}\right]\right. \\
& +\left[\nabla^{i} U_{\lambda}^{*} \nabla_{i} U_{\lambda^{\prime}}\left\langle a_{\lambda}^{\dagger} a_{\lambda^{\prime}}\right\rangle \mathrm{e}^{i\left(\omega_{\lambda}-\omega_{\lambda^{\prime}}\right) t}+\nabla^{i} U_{\lambda} \nabla_{i} U_{\lambda^{\prime}}\left\langle a_{\lambda} a_{\lambda^{\prime}}\right\rangle \mathrm{e}^{-i\left(\omega_{\lambda}+\omega_{\lambda^{\prime}}\right) t}\right] \\
& \left.+m^{2}\left[U_{\lambda}^{*} U_{\lambda^{\prime}}\left\langle a_{\lambda}^{\dagger} a_{\lambda^{\prime}}\right\rangle \mathrm{e}^{i\left(\omega_{\lambda}-\omega_{\lambda^{\prime}}\right) t}+U_{\lambda} U_{\lambda^{\prime}}\left\langle a_{\lambda} a_{\lambda^{\prime}}\right\rangle \mathrm{e}^{-i\left(\omega_{\lambda}+\omega_{\lambda^{\prime}}\right) t}\right]\right\}, \quad(2.6)
\end{aligned}
$$

where $u^{\mu}=\left(\left|g_{t t}\right|^{-1 / 2}, \mathbf{0}\right)$ is the observer's four-velocity, and $U_{\lambda}$ and its derivatives are evaluated at $\mathbf{x}_{\mathbf{0}}$. We have also written $\langle\cdot\rangle \equiv\langle\psi|\cdot| \psi\rangle$ for brevity. Recall that the normal-ordered energy density is the difference between the renormalised energy density in the two states $|\psi\rangle$ and $|0\rangle$.

We now define a weighted energy density

$$
\rho=\int_{-\infty}^{\infty} \mathrm{d} t\left\langle: T_{\mu \nu} u^{\mu} u^{\nu}:\right\rangle f(t)
$$

where $f$ is any smooth, non-negative function decaying at least as fast as $\mathrm{O}\left(t^{-2}\right)$ at infinity, and normalised to have unit integral. Ford and coworkers [10, 11, 12, 13, 15] employ the Lorentzian function (1.1), whose specific properties play a key rôle in their arguments [in particular, the Fourier transform of (1.1) is simply the function $\left.\exp \left(-|\omega| t_{0}\right)\right]$; we emphasise that our arguments apply to general $f$. Substituting from Eq. (2.6), the weighted energy density measured by the observer is

$$
\begin{aligned}
\rho=\operatorname{Re} \sum_{\lambda, \lambda^{\prime}} & \left\{\frac{\omega_{\lambda} \omega_{\lambda^{\prime}}}{\left|g_{t t}\right|}\left[U_{\lambda}^{*} U_{\lambda^{\prime}}\left\langle a_{\lambda}^{\dagger} a_{\lambda^{\prime}}\right\rangle \widehat{f}\left(\omega_{\lambda^{\prime}}-\omega_{\lambda}\right)-U_{\lambda} U_{\lambda^{\prime}}\left\langle a_{\lambda} a_{\lambda^{\prime}}\right\rangle \widehat{f}\left(\omega_{\lambda}+\omega_{\lambda^{\prime}}\right)\right]\right. \\
+ & {\left[\nabla^{i} U_{\lambda}^{*} \nabla_{i} U_{\lambda^{\prime}}\left\langle a_{\lambda}^{\dagger} a_{\lambda^{\prime}}\right\rangle \widehat{f}\left(\omega_{\lambda^{\prime}}-\omega_{\lambda}\right)+\nabla^{i} U_{\lambda} \nabla_{i} U_{\lambda^{\prime}}\left\langle a_{\lambda} a_{\lambda^{\prime}}\right\rangle \widehat{f}\left(\omega_{\lambda}+\omega_{\lambda^{\prime}}\right)\right] }
\end{aligned}
$$




$$
\left.+m^{2}\left[U_{\lambda}^{*} U_{\lambda^{\prime}}\left\langle a_{\lambda}^{\dagger} a_{\lambda^{\prime}}\right\rangle \widehat{f}\left(\omega_{\lambda^{\prime}}-\omega_{\lambda}\right)+U_{\lambda} U_{\lambda^{\prime}}\left\langle a_{\lambda} a_{\lambda^{\prime}}\right\rangle \widehat{f}\left(\omega_{\lambda}+\omega_{\lambda^{\prime}}\right)\right]\right\}
$$

where we define the Fourier transform of $f$ by

$$
\widehat{f}(\omega)=\int_{-\infty}^{\infty} \mathrm{d} t f(t) \mathrm{e}^{-i \omega t} .
$$

By applying the inequality (A.2), proved in the Appendix, to each of the cases $q_{\lambda}=$ $\frac{\omega_{\lambda}}{\left|g_{t t}\right|^{1 / 2}} U_{\lambda}, \nabla_{i} U_{\lambda}$ and $m U_{\lambda}$, we obtain the following manifestly negative lower bound for $\rho$ :

$$
\rho \geq-\frac{1}{2 \pi} \int_{0}^{\infty} \mathrm{d} \omega \sum_{\lambda}\left(\frac{\omega_{\lambda}^{2}}{\left|g_{t t}\right|} U_{\lambda}^{*} U_{\lambda}+\nabla^{i} U_{\lambda}^{*} \nabla_{i} U_{\lambda}+m^{2} U_{\lambda}^{*} U_{\lambda}\right)\left|\widehat{f^{1 / 2}}\left(\omega+\omega_{\lambda}\right)\right|^{2}
$$

Using the field equation satisfied by the spatial mode function [12, 13]:

$$
\nabla^{i} \nabla_{i} U_{\lambda}+\left(\frac{\omega_{\lambda}^{2}}{\left|g_{t t}\right|}-m^{2}\right) U_{\lambda}=0
$$

this inequality can be rewritten as

$$
\rho \geq-\frac{1}{\pi} \int_{0}^{\infty} \mathrm{d} \omega \sum_{\lambda}\left(\frac{\omega_{\lambda}^{2}}{\left|g_{t t}\right|}+\frac{1}{4} \nabla^{i} \nabla_{i}\right)\left|U_{\lambda}\right|^{2}\left|\widehat{f^{1 / 2}}\left(\omega+\omega_{\lambda}\right)\right|^{2} .
$$

This is the desired quantum inequality, which is valid for general sampling functions $f(t)$, subject to the above-stated conditions. Another useful form of it can be obtained by introducing the new variable $u=\omega+\omega_{\lambda}$ :

$$
\rho \geq-\frac{1}{\pi} \int_{\omega_{\min }}^{\infty} \mathrm{d} u\left|\widehat{f^{1 / 2}}(u)\right|^{2} \sum_{\lambda \text { s.t. } \omega_{\lambda} \leq u}\left(\frac{\omega_{\lambda}^{2}}{\left|g_{t t}\right|}+\frac{1}{4} \nabla^{i} \nabla_{i}\right)\left|U_{\lambda}\right|^{2},
$$

with $\omega_{\min } \equiv \min _{\lambda} \omega_{\lambda}$.

To simplify it any further would require a specific choice of $f(t)$. For example, with the even sampling function

$$
f(t)=\frac{2}{\pi} \frac{t_{0}^{3}}{\left(t^{2}+t_{0}^{2}\right)^{2}}
$$

that is peaked at $t=0$, we have

$$
\left|\widehat{f^{1 / 2}}(\omega)\right|^{2}=2 \pi t_{0} \mathrm{e}^{-2|\omega| t_{0}}
$$

In this case, the quantum inequality can be expressed in terms of the Euclidean Green's function

$$
G_{\mathrm{E}}\left(t, \mathbf{x} ; t^{\prime}, \mathbf{x}^{\prime}\right)=\sum_{\lambda} U_{\lambda}^{*}(\mathbf{x}) U_{\lambda}\left(\mathbf{x}^{\prime}\right) \mathrm{e}^{\omega_{\lambda}\left(t-t^{\prime}\right)}
$$


quite compactly as

$$
\rho \geq-\frac{1}{4} \square_{\mathrm{E}} G_{\mathrm{E}}\left(-t_{0}, \mathbf{x} ; t_{0}, \mathbf{x}\right)
$$

where $\square_{\mathrm{E}} \equiv \frac{1}{\left|g_{t t}\right|} \partial_{t_{0}}^{2}+\nabla^{i} \nabla_{i}$ is the Euclidean wave operator. This bound is, in fact, identical to one that was derived in [12, 13] assuming the Lorentzian sampling function (1.1). But because (2.14) is a more sharply peaked function [half the area under the Lorentzian function lies within $|t|<t_{0}$, while this figure is $\frac{1}{2}+\frac{1}{\pi} \simeq 0.82$ for (2.14)], this is a first indication that the inequality derived here is a stronger result.

Finally, we record the fact that for the Lorentzian function,

$$
\left|\widehat{f^{1 / 2}}(\omega)\right|^{2}=\frac{4 t_{0}}{\pi} K_{0}\left(t_{0}|\omega|\right)^{2}
$$

where $K_{0}(x)$ is the modified Bessel function of zeroth order. In the rest of this paper, we shall consider the quantum inequality in specific examples of globally static space-times where the left-hand side of (2.12) or (2.13) can be explicitly evaluated. As these examples have been considered previously by Pfenning and Ford [12, 13, 15], we shall at times be brief and refer to their papers for more details. For the most part we will closely follow their notation and conventions.

\section{Minkowski space}

We begin with a review of the quantum inequality in $n+1$-dimensional Minkowski space, the case that was treated in [14]. The mode functions for a free scalar field of mass $\mu$ are

$$
U_{\mathbf{k}}(\mathbf{x})=\frac{1}{\left[(2 \pi)^{n} 2 \omega_{\mathbf{k}}\right]^{1 / 2}} e^{i \mathbf{k} \cdot \mathbf{x}}, \quad \omega_{\mathbf{k}}=\sqrt{|\mathbf{k}|^{2}+\mu^{2}},
$$

with each component of the $n$-dimensional (spatial) momentum covector $\mathbf{k}$ satisfying $-\infty<$ $k_{i}<\infty$. The quantum inequality (2.12) becomes

$$
\begin{aligned}
\rho & \geq-\frac{1}{2 \pi} \int_{0}^{\infty} \mathrm{d} \omega \int \frac{\mathrm{d}^{n} \mathbf{k}}{(2 \pi)^{n}} \omega_{\mathbf{k}}\left|\widehat{f^{1 / 2}}\left(\omega+\omega_{\mathbf{k}}\right)\right|^{2} \\
& =-\frac{C_{n}}{2 \pi} \int_{0}^{\infty} \mathrm{d} \omega \int_{\mu}^{\infty} \mathrm{d} \omega^{\prime} \omega^{\prime 2}\left(\omega^{\prime 2}-\mu^{2}\right)^{n / 2-1}\left|\widehat{f^{1 / 2}}\left(\omega+\omega^{\prime}\right)\right|^{2}
\end{aligned}
$$

where $C_{n}$ is equal to the area of the unit $n-1$-sphere divided by $(2 \pi)^{n}$, i.e.,

$$
C_{n} \equiv \frac{1}{2^{n-1} \pi^{n / 2} \Gamma\left(\frac{1}{2} n\right)} .
$$


If we make the change of variables $u=\omega+\omega^{\prime}$ and $v=\omega^{\prime}$, the quantum inequality (3.2) can be rewritten as

$$
\rho \geq-\frac{C_{n}}{2 \pi(n+1)} \int_{\mu}^{\infty} \mathrm{d} u\left|\widehat{f^{1 / 2}}(u)\right|^{2} u^{n+1} Q_{n}\left(\frac{u}{\mu}\right),
$$

where the functions $Q_{n}(x)$ are defined by

$$
Q_{n}(x)=(n+1) x^{-(n+1)} \int_{1}^{x} \mathrm{~d} y y^{2}\left(y^{2}-1\right)^{n / 2-1} .
$$

There are several special cases in which this bound can be evaluated analytically [14, notably massless fields in two and four dimensions with the sampling function (1.1). In the former, the bound is four times stronger than that derived by Ford and Roman [11, but $1 \frac{1}{2}$ times weaker than the optimal one of Flanagan [16]. In the latter case, the present bound is $\frac{9}{64}$ of Ford and Roman's result.

\section{Three-dimensional closed universe}

The line element for the static, three-dimensional closed universe is

$$
\mathrm{d} s^{2}=-\mathrm{d} t^{2}+a^{2}\left(\mathrm{~d} \theta^{2}+\sin ^{2} \theta \mathrm{d} \varphi^{2}\right),
$$

where $a$ is the radius of the two-sphere at each constant time-slice, and the angular variables take values $0 \leq \theta \leq \pi, 0 \leq \varphi<2 \pi$ (and will do so for all the space-times considered in this paper). We consider the massive scalar field equation on this background with a coupling of strength $\xi$ to the scalar curvature $R=2 / a^{2}$ :

$$
\square \phi-\left(\mu^{2}+\xi R\right) \phi=0,
$$

whose mode-function solutions are given in terms of the usual spherical harmonics $Y_{l m}(\theta, \varphi)$ by [13, 15

$$
U_{l m}(\mathbf{x})=\frac{1}{\left(2 \omega_{l} a^{2}\right)^{1 / 2}} Y_{l m}(\theta, \varphi),
$$

for $l=0,1,2, \ldots$ and $m=-l,-l+1, \ldots, l$, with

$$
\omega_{l}=a^{-1} \sqrt{l(l+1)+2 \xi+(a \mu)^{2}} .
$$

The $Y_{l m}(\theta, \varphi)$ obey the sum rule

$$
\sum_{m=-l}^{l}\left|Y_{l m}(\theta, \varphi)\right|^{2}=\frac{2 l+1}{4 \pi},
$$


which can be used in (2.12) and (2.13) to show that

$$
\begin{aligned}
\rho & \geq-\frac{1}{8 \pi^{2} a^{2}} \int_{0}^{\infty} \mathrm{d} \omega \sum_{l=0}^{\infty}(2 l+1) \omega_{l}\left|\widehat{f^{1 / 2}}\left(\omega+\omega_{l}\right)\right|^{2} \\
& =-\frac{1}{8 \pi^{2} a^{2}} \int_{\omega_{0}}^{\infty} \mathrm{d} u\left|\widehat{f^{1 / 2}}(u)\right|^{2} \sum_{l=0}^{N(u)}(2 l+1) \omega_{l} .
\end{aligned}
$$

Here, $N(u) \equiv \max \left\{n \in \mathbf{Z}: \omega_{n} \leq u\right\}$, i.e.,

$$
N(u)=\left\lfloor\frac{\sqrt{1-4\left[2 \xi+(a \mu)^{2}-(a u)^{2}\right]}-1}{2}\right\rfloor
$$

where $\lfloor x\rfloor$ denotes the integer part of $x$.

While the bound in (4.6) can be readily evaluated using numerical techniques, it may be worthwhile to first simplify it analytically as much as possible. This may be useful if one should want to draw conclusions about its general properties. In particular, we shall present a general strategy for approximating finite summations like that in (4.6).

The summation in (4.6) can be evaluated using the trapezoidal rule of numerical integration (e.g., see Eq. (3.6.1) of [17]):

$$
\sum_{n=0}^{N} g(n)=\int_{0}^{N} \mathrm{~d} x g(x)+\frac{1}{2}[g(0)+g(N)]+\frac{N}{12} g^{\prime \prime}(\zeta)
$$

for some $\zeta \in(0, N)$. In the present case, $g(x)=(2 x+1) \sqrt{x(x+1)+2 \xi+(a \mu)^{2}}$, and the integral in (4.8) can be evaluated analytically. Furthermore, $g^{\prime \prime}(\zeta)$ is non-decreasing in the interval in question, so its occurrence in (4.6) can be replaced by $g^{\prime \prime}(N)$, at the expense of weakening the bound slightly. We obtain the final inequality

$$
\rho \geq-\frac{1}{8 \pi^{2} a^{3}} \int_{\omega_{0}}^{\infty} \mathrm{d} u\left|\widehat{f^{1 / 2}}(u)\right|^{2}\left\{\int_{0}^{N(u)} \mathrm{d} x g(x)+\frac{1}{2}[g(0)+g(N(u))]+\frac{N(u)}{12} g^{\prime \prime}(N(u))\right\}
$$

with

$$
\begin{aligned}
\int \mathrm{d} x g(x) & =\frac{2}{3}\left[x(x+1)+2 \xi+(a \mu)^{2}\right]^{3 / 2} \\
g^{\prime \prime}(x) & =\frac{3(2 x+1)}{\sqrt{x(x+1)+2 \xi+(a \mu)^{2}}}-\frac{1}{4} \frac{(2 x+1)^{3}}{\left[x(x+1)+2 \xi+(a \mu)^{2}\right]^{3 / 2}} .
\end{aligned}
$$

The graph of the bound in (4.6) is plotted against mass in Fig. 1, for $a=1$ and $\xi=0$. As usual, the sampling function $f(t)$ is taken to be the Lorentzian function (1.1), with $t_{0}=1$. 


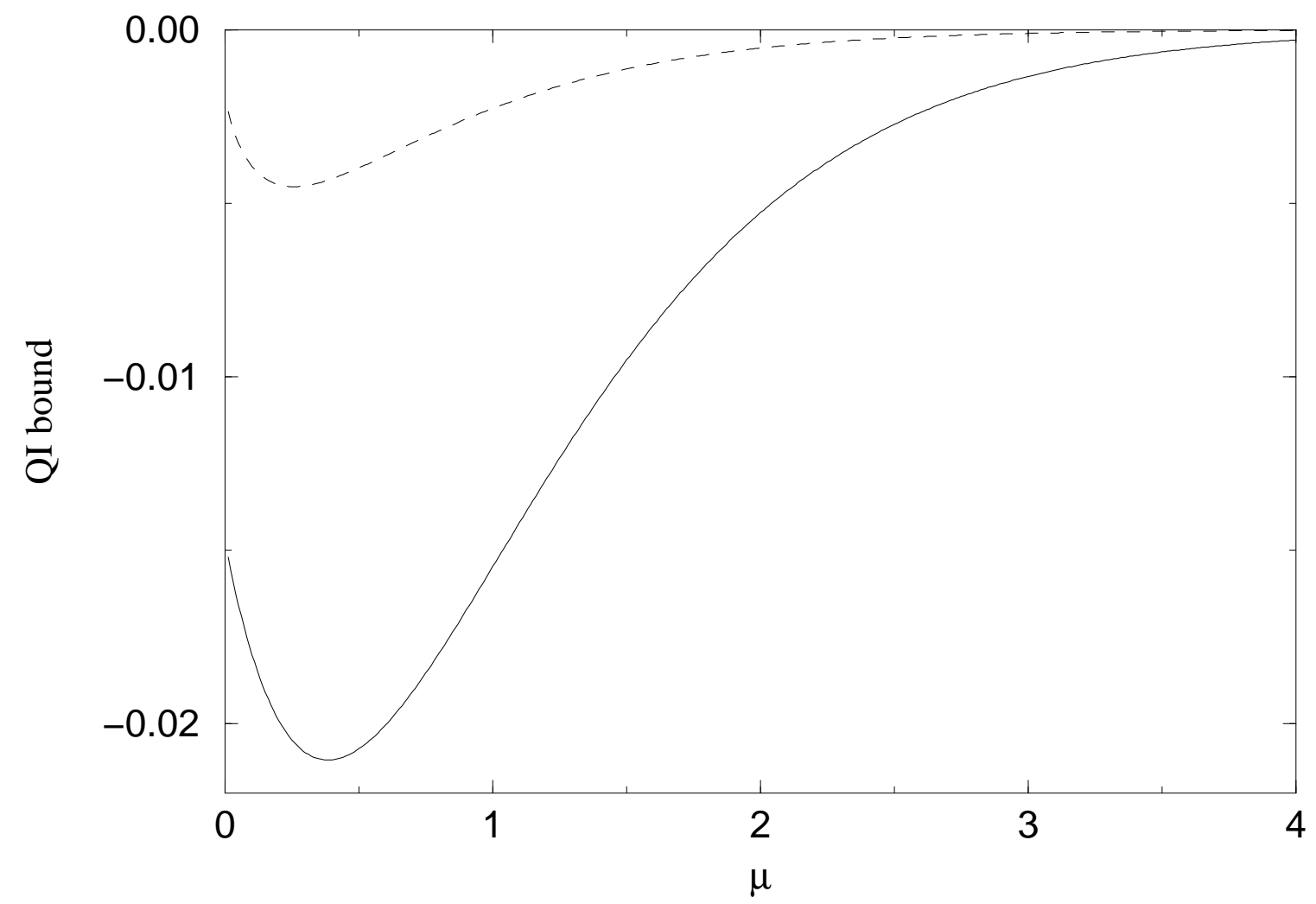

Figure 1: Graph of the QI bound for the 3D closed universe [dashed line], and that obtained by Pfenning and Ford [solid line], against $\mu$.

When plotted on the same scale, that of (4.9) is almost indistinguishable from the former graph. For comparison, the corresponding bound obtained by Pfenning and Ford [13, 15] is also plotted in Fig. 1. It is clear that our bound is stronger for all values of mass.

\section{Four-dimensional Robertson-Walker universe}

We shall first consider the case of the open universe, before proceeding to the closed universe. The line element is

$$
\mathrm{d} s^{2}=-\mathrm{d} t^{2}+a^{2}\left[\mathrm{~d} \chi^{2}+\sinh ^{2} \chi\left(\mathrm{d} \theta^{2}+\sin ^{2} \theta \mathrm{d} \varphi^{2}\right)\right]
$$


where $a$ characterises the scale of the spatial section, and $0 \leq \chi<\infty$. The mode functions for a scalar field of mass $\mu$ are [18]

$$
\begin{aligned}
U_{q l m}(\mathbf{x}) & =\frac{1}{\left(2 \omega_{q} a^{3}\right)^{1 / 2}} \Pi_{q l}^{(-)}(\chi) Y_{l m}(\theta, \varphi), \\
\omega_{q} & =\sqrt{\frac{q^{2}+1}{a^{2}}+\mu^{2}},
\end{aligned}
$$

with $0<q<\infty$ and $l, m$ as usual. The functions $\Pi_{q l}^{-}(\chi)$ satisfy

$$
\Pi_{q l}^{(-)}(\chi) \propto \sinh ^{l} \chi\left(\frac{\mathrm{d}}{\mathrm{d} \cosh \chi}\right)^{l+1} \cos q \chi,
$$

and obey the sum rule

$$
\sum_{l, m}\left|\Pi_{q l}^{(-)}(\chi) Y_{l m}(\theta, \varphi)\right|^{2}=\frac{q^{2}}{2 \pi^{2}} .
$$

The right-hand side does not depend on the angular variables, as is expected of a system with isotropic symmetry. Hence, the quantum inequality (2.12) becomes

$$
\rho \geq-\frac{1}{4 \pi^{3} a^{3}} \int_{0}^{\infty} \mathrm{d} \omega \int_{0}^{\infty} \mathrm{d} q \omega_{q} q^{2}\left|\widehat{f^{1 / 2}}\left(\omega+\omega_{q}\right)\right|^{2} .
$$

Note that this bound is identical in form to that in (four-dimensional) Minkowski space. Both (3.2) and (5.5) can, in fact, be written as

$$
\begin{aligned}
\rho & \geq-\frac{1}{4 \pi^{3}} \int_{0}^{\infty} \mathrm{d} \omega \int_{C}^{\infty} \mathrm{d} \omega^{\prime} \omega^{\prime 2} \sqrt{\omega^{\prime 2}-C^{2}}\left|\widehat{f^{1 / 2}}\left(\omega+\omega^{\prime}\right)\right|^{2} \\
& =-\frac{1}{16 \pi^{3}} \int_{C}^{\infty} \mathrm{d} u\left|\widehat{f^{1 / 2}}(u)\right|^{2} u^{4} Q_{3}\left(\frac{u}{C}\right)
\end{aligned}
$$

where

$$
C \equiv \sqrt{\frac{\epsilon}{a^{2}}+\mu^{2}}, \quad \epsilon= \begin{cases}0 & \text { Minkowski space } \\ 1 & \text { open universe }\end{cases}
$$

and an explicit expression (and graph) for $Q_{3}(x)$ can be found in [14. The Minkowski space result is obviously recovered in the limit of infinite $a$. Furthermore, since $Q_{3}(x)$ is an increasing function on $[1, \infty)$, the bound for general $a$ is tighter than that in Minkowski space for all sampling functions $f(t)$. Pfenning and Ford [13, 15] also noted this for their particular choice of $f(t)$.

We now turn to the closed or Einstein universe, with line element

$$
\mathrm{d} s^{2}=-\mathrm{d} t^{2}+a^{2}\left[\mathrm{~d} \chi^{2}+\sin ^{2} \chi\left(\mathrm{d} \theta^{2}+\sin ^{2} \theta \mathrm{d} \varphi^{2}\right)\right]
$$


where $0 \leq \chi \leq \pi$. The mode functions are 18

$$
\begin{aligned}
U_{n l m}(\mathbf{x}) & =\frac{1}{\left(2 \omega_{n} a^{3}\right)^{1 / 2}} \Pi_{n l}^{(+)}(\chi) Y_{l m}(\theta, \varphi) \\
\omega_{n} & =\sqrt{\frac{n(n+2)}{a^{2}}+\mu^{2}}
\end{aligned}
$$

with $n=0,1,2, \ldots, l=0,1, \ldots, n, m=-l,-l+1 \ldots, l$, and

$$
\Pi_{n l}^{(+)}(\chi) \propto \sin ^{l} \chi\left(\frac{\mathrm{d}}{\mathrm{d} \cos \chi}\right)^{l+1} \cosh (n+1) \chi .
$$

Using the sum rule

$$
\sum_{l, m}\left|\Pi_{n l}^{(+)}(\chi) Y_{l m}(\theta, \varphi)\right|^{2}=\frac{(n+1)^{2}}{2 \pi^{2}},
$$

we obtain the quantum inequality

$$
\begin{aligned}
\rho & \geq-\frac{1}{4 \pi^{3} a^{3}} \int_{0}^{\infty} \mathrm{d} \omega \sum_{n=0}^{\infty} \omega_{n}(n+1)^{2}\left|\widehat{f^{1 / 2}}\left(\omega+\omega_{n}\right)\right|^{2} \\
& =-\frac{1}{4 \pi^{3} a^{3}} \int_{\mu}^{\infty} \mathrm{d} u\left|\widehat{f^{1 / 2}}(u)\right|^{2} \sum_{n=0}^{N(u)} \omega_{n}(n+1)^{2},
\end{aligned}
$$

with

$$
N(u) \equiv\left\lfloor\sqrt{(a u)^{2}-(a \mu)^{2}+1}-1\right\rfloor .
$$

An obvious special case to investigate is $a \mu=1$, in which $\omega_{n}=\mu(n+1)$. The sum in (5.12) may then be evaluated exactly, to give

$$
\rho \geq-\frac{1}{16 \pi^{3} a^{4}} \int_{\mu}^{\infty} \mathrm{d} u\left|\widehat{f^{1 / 2}}(u)\right|^{2}(N(u)+1)^{2}(N(u)+2)^{2} .
$$

This bound may be weakened slightly, by replacing $N(u)=\lfloor a u-1\rfloor$ with the larger quantity $a u-1$, to give

$$
\rho \geq-\frac{1}{16 \pi^{3}} \int_{\mu}^{\infty} \mathrm{d} u\left|\widehat{f^{1 / 2}}(u)\right|^{2}\left(u^{4}+\frac{2 u^{3}}{a}+\frac{u^{2}}{a^{2}}\right)
$$

It clearly differs from the massless Minkowski bound by $\mathrm{O}(1 / a)$ terms. The bounds in (5.14) and (5.15) are plotted in Fig. 8 against mass. The difference between these two graphs can be further minimised using the approximations below, but at the expense of having a more complicated expression for the bound. The corresponding bound derived in [13, 15] is also plotted on the same graph. 


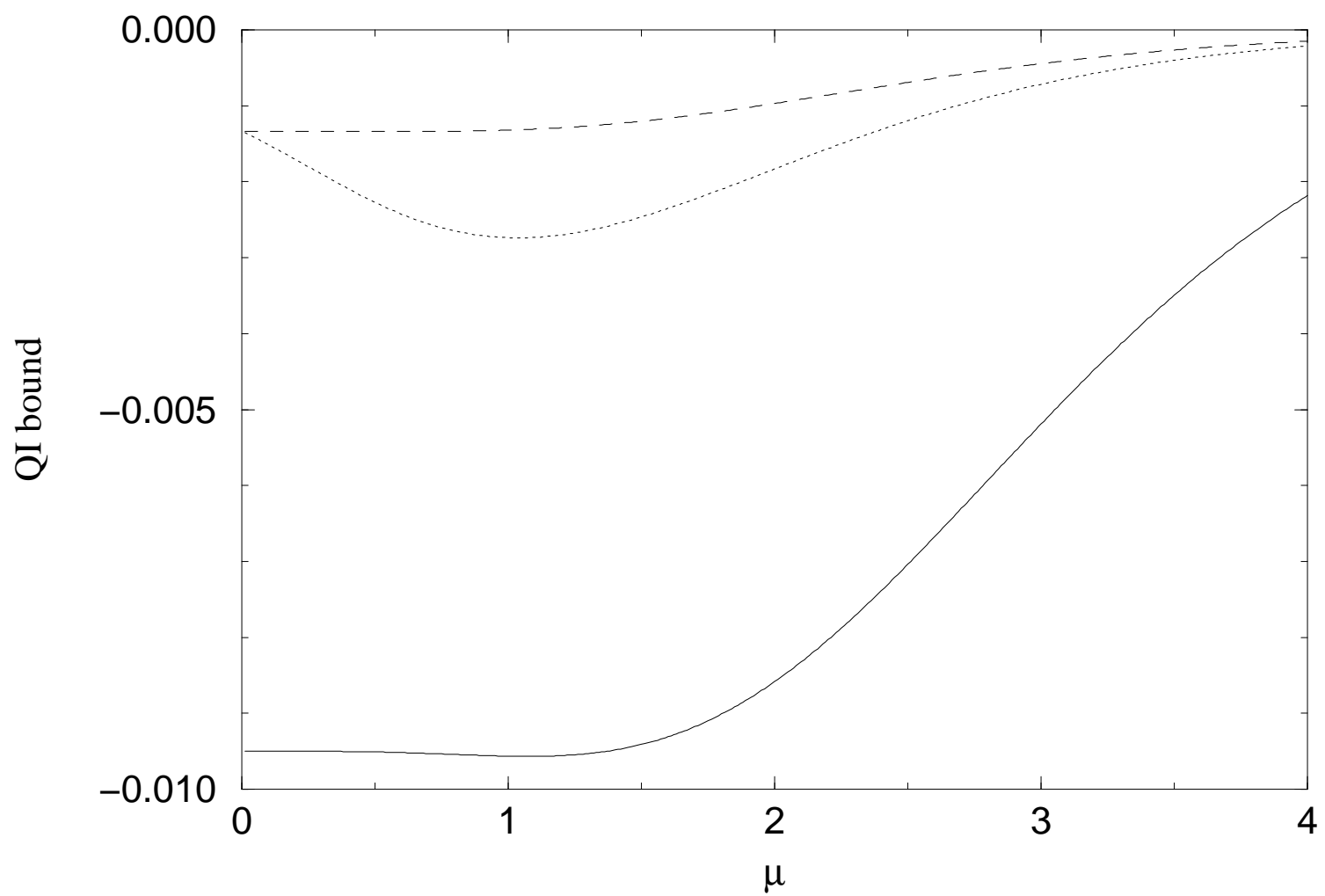

Figure 2: Graphs of the QI bounds for the $4 \mathrm{D}$ closed universe with $a \mu=1$ : (5.14) using a dashed line, (5.15) using a dotted line, and that obtained by Pfenning and Ford [solid line].

Returning to the general case, we note that (5.12) can be written as

$$
\rho \geq-\frac{1}{4 \pi^{3} a^{4}} \int_{\mu}^{\infty} \mathrm{d} u\left|\widehat{f^{1 / 2}}(u)\right|^{2} \sum_{n=1}^{N^{\prime}} n^{2} \sqrt{n^{2}+(a \mu)^{2}-1},
$$

where $N^{\prime} \equiv\left\lfloor a \sqrt{u^{2}-\mu^{2}}\right\rfloor$. The finite sum can again be approximated analytically using the trapezoidal rule, now in the form:

$$
\sum_{n=1}^{N} g(n)=\int_{1}^{N} \mathrm{~d} x g(x)+\frac{1}{2}[g(1)+g(N)]+\frac{N-1}{12} g^{\prime \prime}(\zeta),
$$

for some $\zeta \in(1, N)$. From the fact that the second derivative of $g(x)=x^{2} \sqrt{x^{2}+(a \mu)^{2}-1}$ is non-decreasing in this interval, we obtain the inequality

$$
\rho \geq-\frac{1}{4 \pi^{3} a^{4}} \int_{\mu}^{\infty} \mathrm{d} u\left|\widehat{f^{1 / 2}}(u)\right|^{2}\left\{\int_{1}^{N^{\prime}} \mathrm{d} x g(x)+\frac{1}{2}\left[g(1)+g\left(N^{\prime}\right)\right]+\frac{N^{\prime}-1}{12} g^{\prime \prime}\left(N^{\prime}\right)\right\},
$$




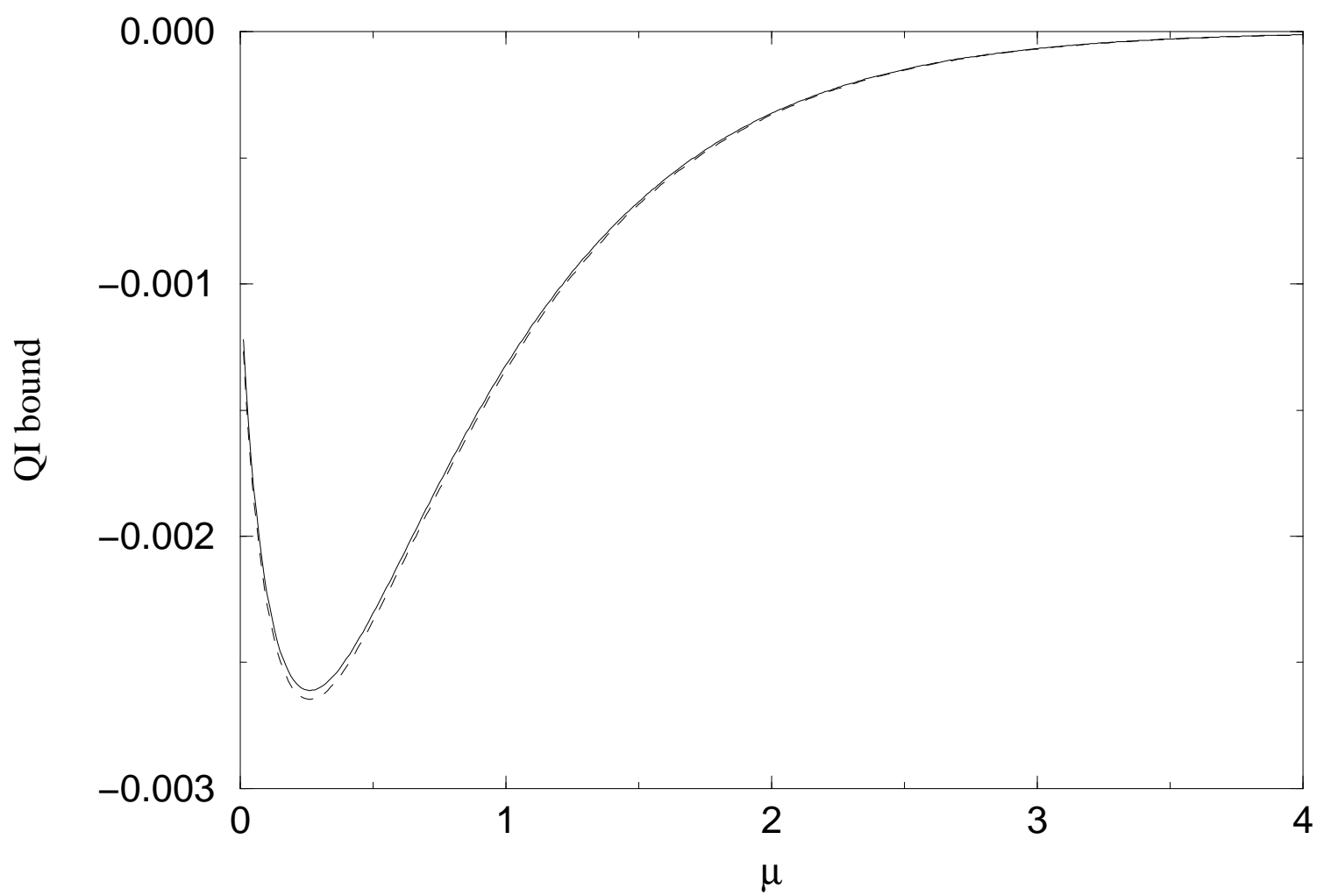

Figure 3: Graphs of the QI bounds for the 4D closed universe: (5.16) using a solid line, and its approximation (5.18) using a dashed line.

with

$$
\begin{aligned}
\int \mathrm{d} x g(x)= & \frac{1}{4} x\left[x^{2}+(a \mu)^{2}-1\right]^{3 / 2}-\frac{1}{8}\left[(a \mu)^{2}-1\right] x \sqrt{x^{2}+(a \mu)^{2}-1} \\
& -\frac{1}{8}\left[(a \mu)^{2}-1\right]^{2} \ln \left(x+\sqrt{x^{2}+(a \mu)^{2}-1}\right) \\
g^{\prime \prime}(x)= & 2 \sqrt{x^{2}+(a \mu)^{2}-1}+\frac{5 x^{2}}{\sqrt{x^{2}+(a \mu)^{2}-1}}-\frac{x^{4}}{\left[x^{2}+(a \mu)^{2}-1\right]^{3 / 2}} .
\end{aligned}
$$

The bound in (5.16) and its approximation in (5.18) are plotted in Fig. 3 against $\mu$, for $a=1$. As can be seen, the approximation is only very slightly weaker than the exact bound. Also plotted in Fig. 1 is the bound obtained in [13, 15], for comparison with (5.16). 


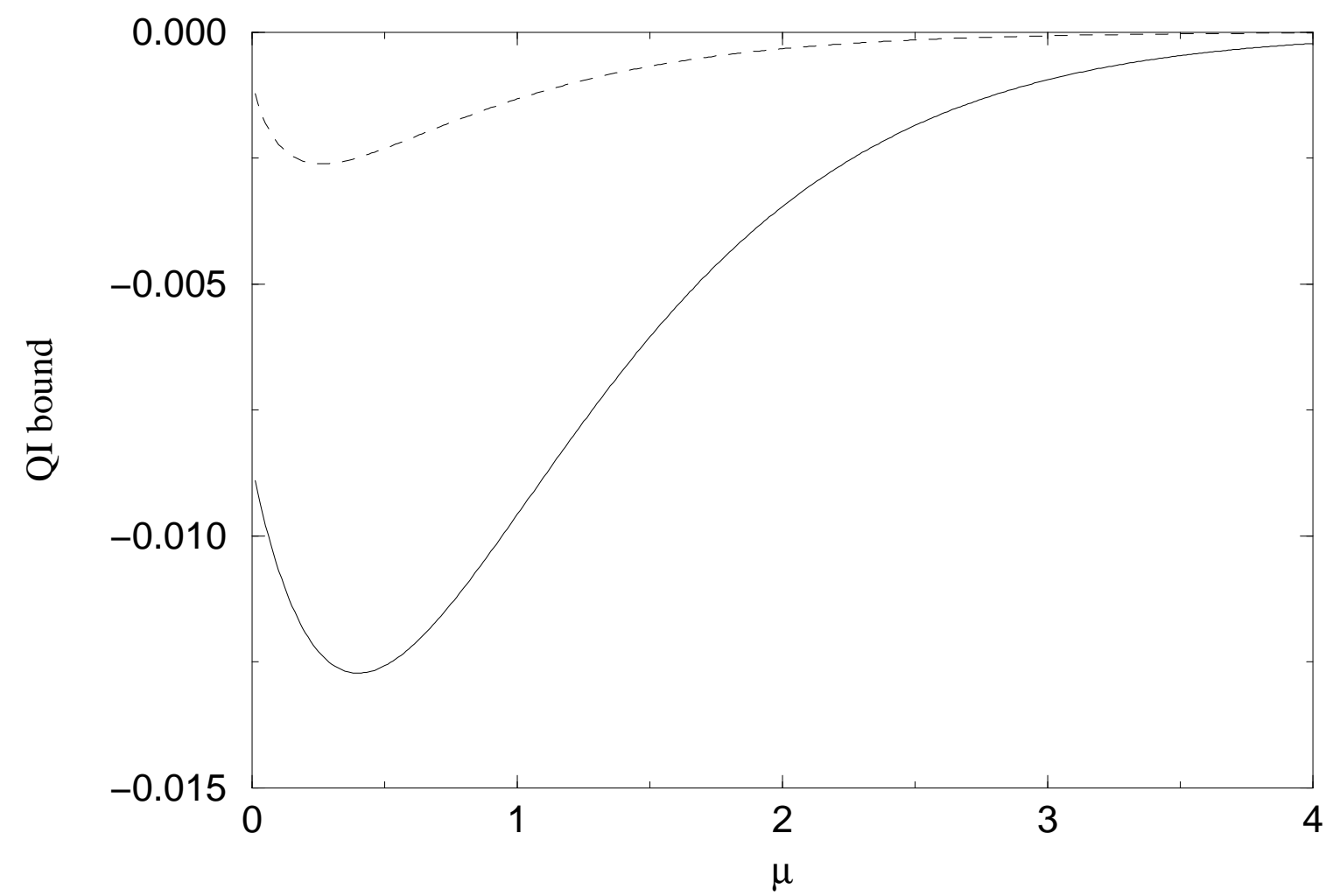

Figure 4: Graphs of the QI bounds for the 4D closed universe: (5.16) using a dashed line, and that of Pfenning and Ford [solid line].

\section{6 de Sitter space-time}

A convenient static parametrisation of the de Sitter universe is

$$
\mathrm{d} s^{2}=-\left(1-\frac{r^{2}}{\alpha^{2}}\right) \mathrm{d} t^{2}+\left(1-\frac{r^{2}}{\alpha^{2}}\right)^{-1} \mathrm{~d} r^{2}+r^{2}\left(\mathrm{~d} \theta^{2}+\sin ^{2} \theta \mathrm{d} \varphi^{2}\right),
$$

with $0 \leq r \leq \alpha$. The surface $r=\alpha$ is the particle horizon for an observer located at the origin. In this representation, the mode functions for a scalar field with mass $\mu$ and energy $\omega$ are

$$
U_{k l m}(\mathbf{x})=\frac{1}{\left(4 \pi \alpha^{2} k\right)^{1 / 2}} f_{k l}(z) Y_{l m}(\theta, \varphi),
$$

where we denote $z \equiv r / \alpha$ and $k \equiv \alpha \omega$. The latter continuously parametrises the mode function from zero to infinity, while $l$ and $m$ are as in Sec. 6 . The radial function can then 
be solved in terms of the hypergeometric function $F(a, b ; c ; z)$ as [19]

$$
f_{k l}(z)=\frac{\Gamma\left(b_{l}^{+}\right) \Gamma\left(b_{l}^{-}\right)}{\Gamma\left(l+\frac{3}{2}\right) \Gamma(i k)} z^{l}\left(1-z^{2}\right)^{i k / 2} F\left(b_{l}^{+}, b_{l}^{-} ; l+\frac{3}{2} ; z^{2}\right),
$$

with

$$
b_{l}^{ \pm} \equiv \frac{1}{2}\left(l+\frac{3}{2}+i k \pm \sqrt{\frac{9}{4}-\alpha^{2} \mu^{2}}\right) .
$$

Using the sum rule (4.5) in the quantum inequality (2.12), we have for an observer at the origin,

$$
\begin{aligned}
\rho \geq- & \frac{1}{64 \pi^{3} \alpha^{4}} \int_{0}^{\infty} \mathrm{d} \omega \int_{0}^{\infty} \mathrm{d} k \sum_{l=0}^{\infty} \frac{2 l+1}{k}\left|\frac{\Gamma\left(b_{l}^{+}\right) \Gamma\left(b_{l}^{-}\right)}{\Gamma\left(l+\frac{3}{2}\right) \Gamma(i k)}\right|^{2} \\
& \lim _{z \rightarrow 0}\left\{\frac{4 k^{2}}{1-z^{2}}+\frac{1}{z^{2}} \partial_{z}\left[z^{2}\left(1-z^{2}\right) \partial_{z}\right]\right\} z^{2 l}\left|F\left(b_{l}^{+}, b_{l}^{-} ; l+\frac{3}{2} ; z^{2}\right)\right|^{2}\left|\widehat{f^{1 / 2}}(\omega+k / \alpha)\right|^{2} .
\end{aligned}
$$

In fact, only the $l=0$ and $l=1$ terms contribute (cf. Eqs. (4.126) and (4.127) of [13]), and the expression may be simplified to give

$$
\begin{aligned}
& \rho \geq-\frac{1}{8 \pi^{5} \alpha^{4}} \int_{0}^{\infty} \mathrm{d} \omega \int_{0}^{\infty} \mathrm{d} k \sinh (\pi k)\left\{\left(k^{2}+\alpha^{2} \mu^{2}\right)\left|\Gamma\left(b_{0}^{+}\right) \Gamma\left(b_{0}^{-}\right)\right|^{2}+4\left|\Gamma\left(b_{1}^{+}\right) \Gamma\left(b_{1}^{-}\right)\right|^{2}\right\} \\
& \times\left|\widehat{f^{1 / 2}}(\omega+k / \alpha)\right|^{2} .
\end{aligned}
$$

As was noted in [12, 13], there are two cases for which the gamma functions in (6.6) can be evaluated analytically, namely when $\mu=0$ and $\sqrt{2} / \alpha$. Assuming the Lorentzian sampling function (1.1) and using (2.18), we obtain, for the massless case,

$$
\rho \geq-\frac{t_{0}}{2 \pi^{4} \alpha^{2}} \int_{0}^{\infty} \mathrm{d} \omega \int_{0}^{\infty} \mathrm{d} \omega^{\prime}\left(5 \omega^{\prime}+2 \alpha^{2} \omega^{\prime 3}\right) K_{0}\left(t_{0}\left(\omega+\omega^{\prime}\right)\right)^{2} .
$$

Defining the new variables $u=\omega+\omega^{\prime}$ and $v=\omega^{\prime}$, the bound becomes

$$
-\frac{t_{0}}{2 \pi^{4} \alpha^{2}} \int_{0}^{\infty} \mathrm{d} u K_{0}\left(t_{0} u\right)^{2} \int_{0}^{u} \mathrm{~d} v\left(5 v+2 \alpha^{2} v^{3}\right) .
$$

This can be explicitly evaluated using the integral

$$
\int_{0}^{\infty} \mathrm{d} u u^{\alpha-1} K_{0}\left(t_{0} u\right)^{2}=\frac{2^{\alpha-3}}{t_{0}^{\alpha} \Gamma(\alpha)} \Gamma\left(\frac{\alpha}{2}\right)^{4},
$$

to obtain

$$
\rho \geq-\frac{3}{32 \pi^{2} t_{0}^{4}} \frac{9}{64}\left[1+\frac{16}{9} \frac{5}{3}\left(\frac{t_{0}}{\alpha}\right)^{2}\right] .
$$




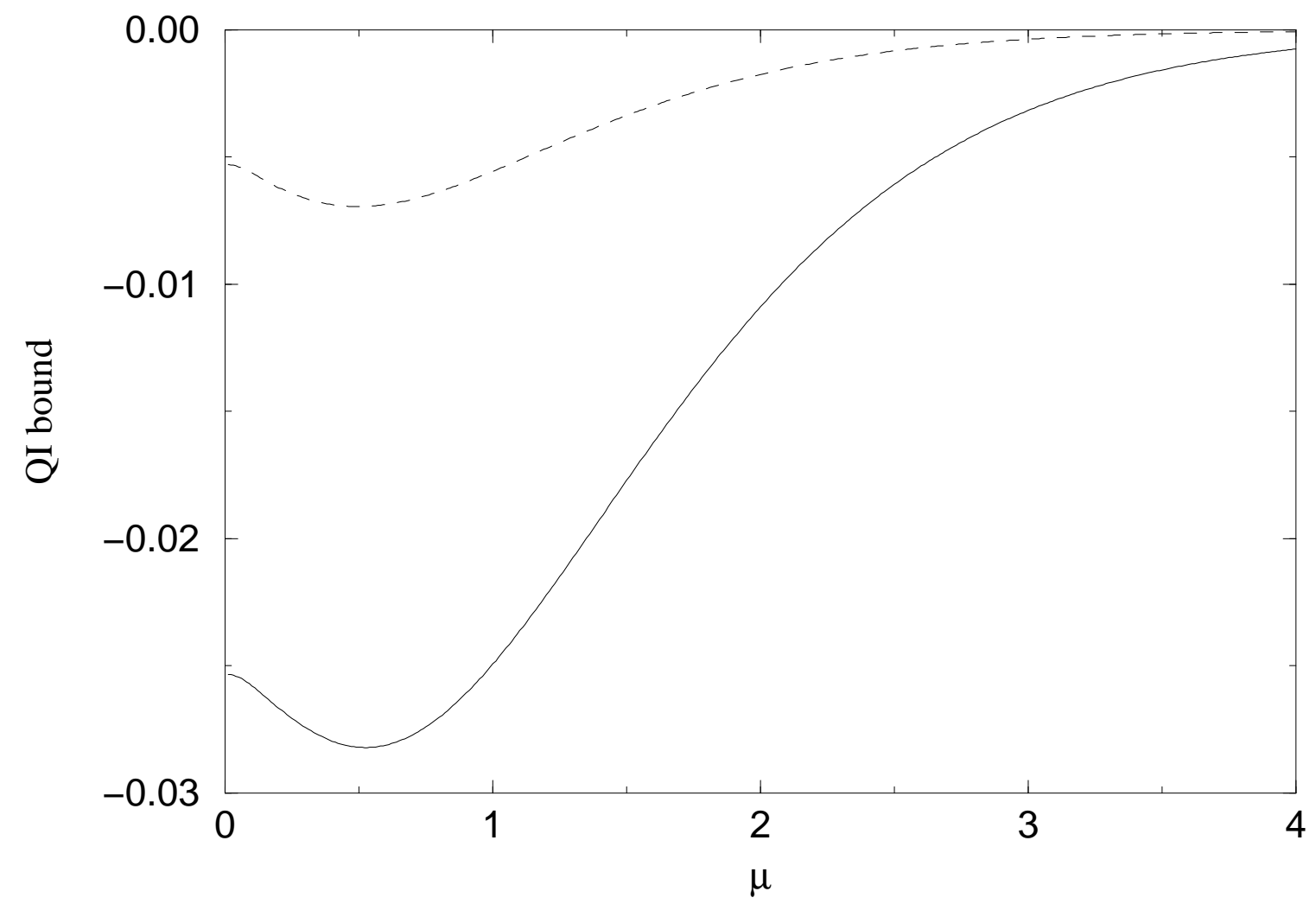

Figure 5: Graphs of the QI bounds for de Sitter space-time: (6.6) using a dashed line, and that of Pfenning and Ford [solid line].

This bound is at least four times stronger than that obtained in [12, [13]:

$$
\rho \geq-\frac{3}{32 \pi^{2} t_{0}^{4}}\left[1+\frac{5}{3}\left(\frac{t_{0}}{\alpha}\right)^{2}\right] .
$$

In the limit $\alpha \rightarrow \infty$ or $t_{0} \rightarrow 0$, we expect to recover the results for Minkowski space. Indeed, the bound in $(\sqrt[6.10]{)})$ is then $\frac{9}{64}$ that in (6.11), as was observed in [14].

When $\mu=\sqrt{2} / \alpha$, we similarly obtain the quantum inequality

$$
\rho \geq-\frac{3}{32 \pi^{2} t_{0}^{4}} \frac{9}{64}\left[1+\frac{16}{9}\left(\frac{t_{0}}{\alpha}\right)^{2}\right],
$$

in contrast to that derived in [12, 13]:

$$
\rho \geq-\frac{3}{32 \pi^{2} t_{0}^{4}}\left[1+\left(\frac{t_{0}}{\alpha}\right)^{2}\right] .
$$


The bound in (6.6) and that derived in [12, 13] are plotted for general $\mu$, and $\alpha=1$, in Fig. 5.

We have, in fact, proved that for general $\mu$, the de Sitter bound (6.6) differs from the Minkowski space bound (3.4) by terms no greater than order $\alpha^{-1 / 2}$ as $\alpha \rightarrow \infty$, and so our results for these cases agree in this limit. This estimate involves bounds on the integrand in Eq. (6.6) which are uniform in $k$ and $\omega$. The proof, which we omit, is accordingly somewhat technical. It is unclear whether the argument can be strengthened to show that the deviation is in fact $\mathrm{O}\left(\alpha^{-2}\right)$ in general, as it is for the specific cases considered in (6.10) and (6.12).

\section{Schwarzschild space-time}

As the final example, we shall examine the quantum inequalities in a black hole space-time. The line element for the Schwarzschild black hole of mass $M$ is

$$
\mathrm{d} s^{2}=-\left(1-\frac{2 M}{r}\right) \mathrm{d} t^{2}+\left(1-\frac{2 M}{r}\right)^{-1} \mathrm{~d} r^{2}+r^{2}\left(\mathrm{~d} \theta^{2}+\sin ^{2} \theta \mathrm{d} \varphi^{2}\right) .
$$

For simplicity, we shall only consider a massless scalar field in this space-time. The mode functions, in the region exterior to the horizon $r>2 M$, take the form [20]

$$
\begin{aligned}
& \vec{U}_{\omega l m}(\mathbf{x})=\frac{1}{(4 \pi \omega)^{1 / 2}} \vec{R}_{l}(\omega \mid r) Y_{l m}(\theta, \varphi) \\
& \overleftarrow{U}_{\omega l m}(\mathbf{x})=\frac{1}{(4 \pi \omega)^{1 / 2}} \overleftarrow{R}_{l}(\omega \mid r) Y_{l m}(\theta, \varphi)
\end{aligned}
$$

where, as usual, $\omega$ is the energy of the field and $Y_{l m}(\theta, \varphi)$ are the spherical harmonics. $\vec{R}_{l}(\omega \mid r)$ and $\overleftarrow{R}_{l}(\omega \mid r)$ are the outgoing and ingoing solutions to the radial part of the wave equation, respectively. Although this equation cannot be solved analytically, the asymptotic forms of the solutions are known near the horizon and at infinity.

Again, using the sum rule (4.5), we see that the quantum inequality (2.12) becomes

$$
\begin{aligned}
& \rho \geq-\frac{1}{16 \pi^{3}} \int_{0}^{\infty} \mathrm{d} \omega \int_{0}^{\infty} \frac{\mathrm{d} \omega^{\prime}}{\omega^{\prime}} \sum_{l=0}^{\infty}(2 l+1)\left\{\frac{\omega^{\prime 2}}{1-\frac{2 M}{r}}+\frac{1}{4 r^{2}} \partial_{r}\left[r^{2}(1-2 M / r) \partial_{r}\right]\right\} \\
& \times\left[\left|\vec{R}_{l}\left(\omega^{\prime} \mid r\right)\right|^{2}+\left|\overleftarrow{R}_{l}\left(\omega^{\prime} \mid r\right)\right|^{2}\right]\left|\widehat{f^{1 / 2}}\left(\omega+\omega^{\prime}\right)\right|^{2}
\end{aligned}
$$

In writing this, we are assuming that the mode functions are defined to have positive frequency with respect to the time-like Killing vector $\partial_{t}$. This is the Boulware vacuum. Now, 
in the two regions where $\vec{R}_{l}(\omega \mid r)$ and $\overleftarrow{R}_{l}(\omega \mid r)$ are known explicitly, we have 21]

$$
\sum_{l=0}^{\infty}(2 l+1)\left|\vec{R}_{l}(\omega \mid r)\right|^{2} \simeq \begin{cases}4 \omega^{2}(1-2 M / r)^{-1}, & r \rightarrow 2 M \\ \frac{1}{r^{2}} \sum_{l=0}^{\infty}(2 l+1)\left|B_{l}(\omega)\right|^{2}, & r \rightarrow \infty\end{cases}
$$

and

$$
\sum_{l=0}^{\infty}(2 l+1)\left|\overleftarrow{R}_{l}(\omega \mid r)\right|^{2} \simeq \begin{cases}\frac{1}{4 M^{2}} \sum_{l=0}^{\infty}(2 l+1)\left|B_{l}(\omega)\right|^{2}, & r \rightarrow 2 M \\ 4 \omega^{2}, & r \rightarrow \infty\end{cases}
$$

If we further assume the low-energy condition $2 M \omega \ll 1$, then 22]

$$
B_{l}(\omega) \simeq \frac{(l !)^{3}}{(2 l+1) !(2 l) !}(-4 i M \omega)^{l+1} .
$$

These results can be substituted into (7.3), and the bound explicitly evaluated using the integral (6.9). However, the maximum value of $l$ for which the expansion in (7.3) is valid depends on the order of the leading terms which have been dropped in $B_{l}(\omega)$. If (7.6) is exact to $\mathrm{O}\left[(M \omega)^{l+2}\right]$, then only the $l=0$ terms should be retained, as the $B_{1}$ contribution would be smaller than the corrections to $B_{0}$ [12, 13.

Near the horizon, the quantum inequality can be expressed in terms of the observer's proper time:

$$
\tau_{0}=\left(1-\frac{2 M}{r}\right)^{\frac{1}{2}} t_{0}
$$

as

$$
\rho \geq-\frac{3}{32 \pi^{2} \tau_{0}^{4}}\left\{\frac{1}{24}\left(\frac{2 M \tau_{0}}{r^{2}}\right)^{2}\left(1-\frac{2 M}{r}\right)^{-1}+\frac{9}{64}\left[1+\left(1-\frac{2 M}{r}\right)\right]+\cdots\right\},
$$

where the ellipsis denotes higher-order terms that have been dropped. This is to be compared with the result derived in 12, 13]:

$$
\rho \geq-\frac{3}{32 \pi^{2} \tau_{0}^{4}}\left\{\frac{1}{6}\left(\frac{2 M \tau_{0}}{r^{2}}\right)^{2}\left(1-\frac{2 M}{r}\right)^{-1}+1+\left(1-\frac{2 M}{r}\right)+\cdots\right\} .
$$

The bound in (7.8) is between $\frac{9}{64}$ and $\frac{1}{4}$ that in (7.9), at least in the present approximation. Note that in either case, the bound becomes arbitrarily negative near the horizon of the black hole.

On the other hand, the quantum inequality for an observer near infinity becomes

$$
\rho \geq-\frac{3}{32 \pi^{2} \tau_{0}^{4}} \frac{9}{64}\left\{1-\frac{2 M}{r}+\left(\frac{2 M}{r}\right)^{2}\left[1+\frac{16}{9} \frac{1}{3}\left(\frac{\tau_{0}}{r}\right)^{2}\right]-\left(\frac{2 M}{r}\right)^{3}\left[1+\frac{16}{9}\left(\frac{\tau_{0}}{r}\right)^{2}\right]+\cdots\right\}
$$


while the corresponding inequality obtained in $[12$, 13] is

$$
\rho \geq-\frac{3}{32 \pi^{2} \tau_{0}^{4}}\left\{1-\frac{2 M}{r}+\left(\frac{2 M}{r}\right)^{2}\left[1+\frac{1}{3}\left(\frac{\tau_{0}}{r}\right)^{2}\right]-\left(\frac{2 M}{r}\right)^{3}\left[1+\left(\frac{\tau_{0}}{r}\right)^{2}\right]+\cdots\right\} .
$$

Again, the former bound is between $\frac{9}{64}$ and $\frac{1}{4}$ the latter. It gives the correct Minkowski space result in the limit $r \rightarrow \infty$ or $M \rightarrow 0$.

\section{Concluding remarks}

In summary, we have derived new quantum inequalities (2.12) or (2.13) on the normal-ordered averaged energy density in static space-times, that are valid for quite general sampling functions. They were then applied to several standard examples using the Lorentzian sampling function. (Of course, other space-times could readily be considered, such as Rindler space, flat space with perfectly reflecting mirrors, and other black holes [12, 13.) The resulting bounds are stronger than previous results, and would lead to even tighter constraints on the various exotic space-times mentioned at the beginning of the paper. Before we conclude, a few comments are in order.

An important question is whether our quantum inequalities are optimal. This could, for example, be proved by finding a quantum state that saturates the bound, which would necessarily belong to the kernel of all the operators $\mathcal{O}^{ \pm}(\omega)$ in (A.6). However, it is known that our bound, when applied to a massless scalar field in two-dimensional Minkowski space, is $1 \frac{1}{2}$ times weaker than the optimal value obtained by Flanagan [16]. Unfortunately, his derivation relies on some special features of two-dimensional massless field theory, and does not appear to generalise to other more realistic cases.

An interesting application of our quantum inequality would be to the static MorrisThorne-type wormholes [1]. Ford and Roman have applied the flat-space version of their quantum inequalities to this case, and have found that they constrain the size of such wormholes [5]. They justified this procedure by making the sampling timescale much shorter than the minimum characteristic curvature scale, so that space-time appears locally flat. However, it would be desirable to verify this calculation using the full curved space results; this should not be too difficult once the form of the scalar field mode functions in the wormhole space-time have been determined. 


\section{Acknowledgment}

CJF thanks Simon Eveson for useful discussions concerning the use of the trapezoidal rule in Secs. 4 and 5 .

\section{A Appendix}

The inequality needed to prove the results in Sec. 2 is a generalisation of one which was first derived in [14 using the convolution theorem. Suppose $f$ is a smooth, non-negative function of $t$, decaying at least as fast as $\mathrm{O}\left(t^{-2}\right)$ for $t \rightarrow \pm \infty$. Let operators $S^{ \pm}$be defined by

$$
S^{ \pm}=\operatorname{Herm} \sum_{\lambda, \lambda^{\prime}}\left\{\widehat{f}\left(\omega_{\lambda^{\prime}}-\omega_{\lambda}\right) \overline{q_{\lambda}} q_{\lambda^{\prime}} a_{\lambda}^{\dagger} a_{\lambda^{\prime}} \pm \widehat{f}\left(\omega_{\lambda}+\omega_{\lambda^{\prime}}\right) q_{\lambda^{\prime}} q_{\lambda} a_{\lambda^{\prime}} a_{\lambda}\right\}
$$

where the $q_{\lambda}$ are complex coefficients, 2] and Herm $X \equiv \frac{1}{2}\left(X+X^{\dagger}\right)$ is the Hermitian part of an operator $X$. We will show that the expectation values $\left\langle S^{ \pm}\right\rangle$obey

$$
\left\langle S^{ \pm}\right\rangle \geq-\frac{1}{2 \pi} \int_{0}^{\infty} \mathrm{d} \omega \sum_{\lambda}\left|\widehat{f^{1 / 2}}\left(\omega+\omega_{\lambda}\right)\right|^{2}\left|q_{\lambda}\right|^{2},
$$

in any normalised quantum state, where $f^{1 / 2}(t) \equiv \sqrt{f(t)}$ is the pointwise square-root of $f(t)$.

To obtain this result, first define a function $g$ by

$$
g(\omega)=\frac{1}{\sqrt{2 \pi}} \widehat{f^{1 / 2}}(\omega) .
$$

We have $\overline{g(\omega)}=g(-\omega)$ because $f^{1 / 2}$ is real, and

$$
(g \star g)(\omega)=\widehat{f}(\omega)
$$

by the convolution theorem, where $\star$ is given by

$$
\left(g_{1} \star g_{2}\right)(\omega)=\int_{-\infty}^{\infty} \mathrm{d} \omega^{\prime} g_{1}\left(\omega-\omega^{\prime}\right) g_{2}\left(\omega^{\prime}\right)
$$

Next, define the following operators on the space of quantum states:

$$
\mathcal{O}^{ \pm}(\omega)=\sum_{\lambda}\left\{\overline{g\left(\omega-\omega_{\lambda}\right)} q_{\lambda} a_{\lambda} \pm \overline{g\left(\omega+\omega_{\lambda}\right)} \overline{q_{\lambda}} a_{\lambda}^{\dagger}\right\}
$$

\footnotetext{
${ }^{2}$ For clarity, we shall denote complex conjugation by an overline in this Appendix.
} 
Using the canonical commutation relations (2.5) and symmetrising in $\lambda, \lambda^{\prime}$, we obtain

$$
\begin{aligned}
\int_{0}^{\infty} \mathrm{d} \omega \mathcal{O}^{ \pm}(\omega)^{\dagger} \mathcal{O}^{ \pm}(\omega)=\int_{0}^{\infty} \mathrm{d} \omega \sum_{\lambda, \lambda^{\prime}}\{ & g\left(\omega-\omega_{\lambda}\right) \overline{g\left(\omega-\omega_{\lambda^{\prime}}\right)} \overline{q_{\lambda}} q_{\lambda^{\prime}} a_{\lambda}^{\dagger} a_{\lambda^{\prime}} \\
& +g\left(\omega+\omega_{\lambda}\right) \overline{g\left(\omega+\omega_{\lambda^{\prime}}\right)} q_{\lambda} \overline{q_{\lambda^{\prime}}} a_{\lambda} a_{\lambda^{\prime}}^{\dagger} \\
& \pm g\left(\omega-\omega_{\lambda}\right) \overline{g\left(\omega+\omega_{\lambda^{\prime}}\right)} \overline{q_{\lambda} q_{\lambda^{\prime}}} a_{\lambda_{\lambda}}^{\dagger} a_{\lambda^{\prime}}^{\dagger} \\
& \left. \pm g\left(\omega+\omega_{\lambda}\right) \overline{g\left(\omega-\omega_{\lambda^{\prime}}\right)} q_{\lambda} q_{\lambda^{\prime}} a_{\lambda} a_{\lambda^{\prime}}\right\} \\
=S^{ \pm}+\mathbb{1} \int_{0}^{\infty} & \mathrm{d} \omega \sum_{\lambda}\left|g\left(\omega+\omega_{\lambda}\right)\right|^{2}\left|q_{\lambda}\right|^{2},
\end{aligned}
$$

where $S^{ \pm}$are given by

$$
S^{ \pm}=\operatorname{Herm} \sum_{\lambda, \lambda^{\prime}}\left\{F\left(\omega_{\lambda}, \omega_{\lambda^{\prime}}\right) \overline{q_{\lambda}} q_{\lambda^{\prime}} a_{\lambda}^{\dagger} a_{\lambda^{\prime}} \pm G\left(\omega_{\lambda}, \omega_{\lambda^{\prime}}\right) q_{\lambda^{\prime}} q_{\lambda} a_{\lambda^{\prime}} a_{\lambda}\right\}
$$

and $F, G$ are

$$
\begin{aligned}
F\left(\omega_{\lambda}, \omega_{\lambda^{\prime}}\right) & =\int_{0}^{\infty} \mathrm{d} \omega\left\{g\left(\omega-\omega_{\lambda}\right) \overline{g\left(\omega-\omega_{\lambda^{\prime}}\right)}+\overline{g\left(\omega+\omega_{\lambda}\right)} g\left(\omega+\omega_{\lambda^{\prime}}\right)\right\} \\
& =\int_{0}^{\infty} \mathrm{d} \omega\left\{g\left(\omega-\omega_{\lambda}\right) g\left(-\omega+\omega_{\lambda^{\prime}}\right)+g\left(-\omega-\omega_{\lambda}\right) g\left(\omega+\omega_{\lambda^{\prime}}\right)\right\} \\
& =\int_{-\infty}^{\infty} \mathrm{d} \omega g\left(\omega_{\lambda^{\prime}}-\omega\right) g\left(\omega-\omega_{\lambda}\right) \\
& =(g \star g)\left(\omega_{\lambda^{\prime}}-\omega_{\lambda}\right)=\widehat{f}\left(\omega_{\lambda^{\prime}}-\omega_{\lambda}\right), \\
G\left(\omega_{\lambda}, \omega_{\lambda^{\prime}}\right) & =\int_{0}^{\infty} \mathrm{d} \omega\left\{g\left(\omega+\omega_{\lambda}\right) \overline{g\left(\omega-\omega_{\lambda^{\prime}}\right)}+\overline{g\left(\omega-\omega_{\lambda}\right)} g\left(\omega+\omega_{\lambda^{\prime}}\right)\right\} \\
& =\int_{0}^{\infty} \mathrm{d} \omega\left\{g\left(\omega+\omega_{\lambda}\right) g\left(\omega_{\lambda^{\prime}}-\omega\right)+g\left(\omega_{\lambda}-\omega\right) g\left(\omega+\omega_{\lambda^{\prime}}\right)\right\} \\
& =\int_{-\infty}^{\infty} \mathrm{d} \omega g\left(\omega+\omega_{\lambda}\right) g\left(\omega_{\lambda^{\prime}}-\omega\right) \\
& =(g \star g)\left(\omega_{\lambda}+\omega_{\lambda^{\prime}}\right)=\widehat{f}\left(\omega_{\lambda}+\omega_{\lambda^{\prime}}\right) .
\end{aligned}
$$

The final equalities show that (A.8) agrees with the definition (A.1).

Since the left-hand side of (A.7) is manifestly non-negative, we conclude that the expectation value of $S^{ \pm}$in any normalised quantum state must satisfy the inequality

$$
\left\langle S^{ \pm}\right\rangle \geq-\int_{0}^{\infty} \mathrm{d} \omega \sum_{\lambda}\left|g\left(\omega+\omega_{\lambda}\right)\right|^{2}\left|q_{\lambda}\right|^{2},
$$

which is the desired result (A.2). Note that the inequality proved in [14] corresponds to the special case where the $q_{\lambda}$ are real and $f$ is an even function of $t$. 


\section{References}

[1] M.S. Morris and K.S. Thorne, Am. J. Phys. 56, 395 (1988).

[2] M.S. Morris, K.S. Thorne, and U. Yurtsever, Phys. Rev. Lett. 61, 1446 (1988).

[3] M. Alcubierre, Class. Quantum Grav. 11, L73 (1994).

[4] S.V. Krasnikov, Phys. Rev. D 57, 4760 (1998) gr-qc/9511068.

[5] L.H. Ford and T.A. Roman, Phys. Rev. D 53, 5496 (1996) gr-qc/9510071.

[6] M.J. Pfenning and L.H. Ford, Class. Quantum Grav. 14, 1743 (1997) gr-qc/9702026.

[7] A.E. Everett and T.A. Roman, Phys. Rev. D 56, 2100 (1997) gr-qc/9702049.

[8] K.D. Olum, Phys. Rev. Lett. 81, 3567 (1998) gr-qc/9805003.

[9] H. Epstein, V. Glaser, and A. Jaffe, Nuovo Cimento 36, 1016 (1965).

[10] L.H. Ford and T.A. Roman, Phys. Rev. D 51, 4277 (1995) gr-qc/9410043.

[11] L.H. Ford and T.A. Roman, Phys. Rev. D 55, 2082 (1997) gr-qc/9607003.

[12] M.J. Pfenning and L.H. Ford, Phys. Rev. D 57, 3489 (1998) gr-qc/9710055.

[13] M.J. Pfenning, 'Quantum inequality restrictions on negative energy densities in curved spacetimes,' Ph.D. Thesis, gr-qc/9805037.

[14] C.J. Fewster and S.P. Eveson, Phys. Rev. D 58, 084010 (1998) gr-qc/9805024.

[15] M.J. Pfenning and L.H. Ford, Phys. Rev. D 55, 4813 (1997) gr-qc/9608005.

[16] E.E. Flanagan, Phys. Rev. D 56, 4922 (1997) gr-qc/9706006.

[17] F.B. Hildebrand, Introduction to Numerical Analysis 2nd ed. (Tata McGraw-Hill, New Delhi, 1974).

[18] L. Parker and S.A. Fulling, Phys. Rev. D 9, 341 (1974).

[19] A. Higuchi, Class. Quantum Grav. 4, 721 (1987). 
[20] B.S. DeWitt, Phys. Rep. 19, 295 (1975).

[21] P. Candelas, Phys. Rev. D 21, 2185 (1980).

[22] B.P. Jensen, J.G. McLaughlin, and A.C. Ottewill, Phys. Rev. D 45, 3002 (1992). 